\title{
Parity anomalies on 4D manifolds with boundaries
}

\author{
Maxim Kurkov ${ }^{1,2, *}$ \\ ${ }^{1}$ Dipartimento di Fisica "Ettore Pancini”, Università di Napoli Federico II, Napoli, Complesso Univ. \\ Monte S. Angelo, Via Cintia, I-80126 Napoli, Italy \\ ${ }^{2}$ INFN, Sezione di Napoli, Via Cintia, 80126 Napoli, Italy
}

Abstract. We discuss a parity breaking in theories of fermions, which are trapped inside four-manifolds with boundaries. Even though these theories are parity-invariant at the classical level, the radiative corrections induce parityviolating boundary terms. The effect is present in both gauge and gravitational sectors.

\section{Introduction}

In this article we review the recent results $[1,2]$ concerning parity anomalies in quantum field theories (QFT) on four-dimensional manifolds with boundaries.

Parity anomalies were discovered in the context of massless gauge theories on odddimensional manifolds without boundaries $[3,4]$. Let us briefly recall the story. Consider three-dimensional massless fermions $\psi$, which propagate in a gauge background $A$. Classical Dirac equations of motion remain invariant upon the parity transformation:

$$
\mathrm{P}_{3 \mathrm{~d}}: \quad\left\{\begin{array}{l}
\psi(x) \longrightarrow \psi(-x) \\
A_{i} \longrightarrow-A_{i}(-x)
\end{array}, \quad i=0, ., 2,\right.
$$

however, the one-loop quantum correction to the effective action $W$ breaks this symmetry due to a presence of the Chern-Simons term:

$$
W[A]= \pm \frac{\mathrm{i}}{8 \pi} \int d^{3} x A_{i} \partial_{j} A_{k} \epsilon^{i j k}+\ldots
$$

where "..." stands for parity-even contributions.

Later on these results were generalised to a gravitational sector [18-20]. Nowadays parity anomalies attract significant attention in a relation to various areas of theoretical physics: topological insulators (and other topological materials) [5-9], Casimir effect [10], dualities $[11,12]$ and domain walls $[13,14]$.

Let us track the origin of this anomaly. The one-loop effective action is (formally) given by a determinant of the Dirac operator

$$
e^{-W[A]}=\operatorname{det}(\not D[A]), \quad \not D[A] \equiv \mathrm{i} \gamma^{i}\left(\frac{\partial}{\partial x^{i}}+\mathrm{i} A_{i}(x)\right) .
$$

\footnotetext{
*e-mail: max.kurkov@gmail.com
} 
The parity transformation (1) reflects the spectrum of the Dirac operator:

$$
\mathrm{P}_{3 \mathrm{~d}}: \quad(\not D[A] \psi)(x)=\lambda \psi(x) \longrightarrow(-\not D[A] \psi)(-x)=\lambda \psi(-x),
$$

therefore the parity anomaly expresses a spectral asymmetry:

$$
W_{\text {odd }}=-\frac{1}{2}(\log \operatorname{det}(\not D[A])-\log \operatorname{det}(-\not D[A])) \text {. }
$$

In this paper we consider a 4-dimensional QFT, where Dirac fermions "live" inside the manifold $\mathcal{M}$ of a finite size and can not penetrate through the boundary $\partial \mathcal{M}$. Theories with boundaries arise in the context of BCFT, for the recent progress see e.g. [15-17] and refs therein. We will demonstrate that existence of a boundary creates a spectral asymmetry, which results in a presence of parity-violating boundary terms in the one-loop effective action.

For the sake of simplicity we consider a massless abelian and Euclidean QFT on a compact manifold. This paper is organised as follows. In Sec. 2 we formalise the notion of confined fermions, imposing the local bag boundary conditions. In Sec. 3 we carefully define the 4-d parity anomaly, imposing the zeta-function regularisation of the fermionic determinant. Sec. 4 is devoted to a computation of the gauge contribution to the parity anomaly. In Sec. 5 the gravitational contribution is discussed. In Sec. 6 we comment on the underlying classical symmetry which is violated by quantum corrections. We round up with the discussions and the outlook in Sec. 7.

\section{Confined fermions in 4D and boundary conditions}

Let us describe our setup more precisely. The Dirac operator is the usual one

$$
\not D=\mathrm{i} \gamma^{\mu}\left(\nabla_{\mu}+\mathrm{i} A_{\mu}\right), \quad \nabla_{\mu} \equiv \partial_{\mu}+\omega_{\mu}, \quad \mu=0, . ., 3,
$$

where $A$ and $\omega$ stand for the (abelian) gauge and the spin connections respectively. The manifold $\mathcal{M}$, which represents the space-time, is not supposed to be flat.

A presence of the boundary $\partial \mathcal{M}=\cup_{\alpha}(\partial \mathcal{M})_{\alpha}$ requires a specification of boundary conditions. Hereafter $\alpha$ labels different connected components of the boundary. It is natural to require that:

$$
\left.*) \not D^{\dagger}=\not D, \quad * *\right)\left.\left(\bar{\psi} \gamma^{\mu} \psi\right) n_{\mu}\right|_{\partial \mathcal{M}}=0
$$

where $n$ stands for the the inward pointing unit normal vector to the boundary. The former (self-adjointness) requirement allows to manipulate with the spectrum of the Euclidean Dirac operator and to define a regularised determinant of the Dirac operator using spectral techniques. The latter requirement has a transparent physical meaning: the current through the boundary vanishes, i.e. our fermions are confined inside the manifold $\mathcal{M}$.

The (Euclidean version of) the MIT bag boundary conditions fulfill both requirements. For each component $\partial \mathcal{M}_{\alpha}$ of the boundary we define the projectors

$$
\Pi_{ \pm}=\frac{1}{2}\left(\mathbf{1} \pm \mathrm{i} \epsilon_{\alpha} \gamma^{5} \gamma^{n}\right), \quad \epsilon_{\alpha} \in\{-1,+1\}
$$

and impose

$$
\left.\Pi_{-} \psi\right|_{\partial \mathcal{M}}=0
$$




\section{Parity anomaly in 4D: the definition}

A parity anomaly can be defined in arbitrary dimension via Eq. (5), however, strictly speaking, this expression does not make any sense, unless one introduces the regularisation. Below we work with the $\zeta$-function regularisation:

$$
\text { Reg. : } \quad W[\not D] \equiv-\log \operatorname{det}(\not D[A]) \longrightarrow \mu^{s} \Gamma(s) \zeta(s, \not D) \equiv W_{s}[\not D] .
$$

In this formula $\mu$ stands for an arbitrary constant of the dimension of mass, and the zeta function of the Dirac operator is defined in the following way:

$$
\zeta(s, \not D)=\sum_{\lambda>0} \lambda^{-s}+e^{-\mathrm{i} \pi s} \sum_{\lambda<0}(-\lambda)^{-s},
$$

where the eigenvalues of $\not D$ are denoted through $\lambda$. The sums in (10) are convergent at $\mathfrak{R} s>4$ and from this region can be analitically continued as a meromorphic function to the whole complex plane. This regularisation is removed at the physical limit $s \rightarrow 0$.

The parity-odd contribution to the $\zeta$ function can be rewritten in terms of the $\eta$ function

$$
\zeta_{\text {odd }}(s, \not D) \equiv \frac{1}{2}\left(\zeta_{\text {odd }}(s, \not D)-\zeta_{\text {odd }}(s,-\not D)\right)=\frac{1}{2}\left(1-e^{-\mathrm{i} \pi s}\right) \eta(s, \not D),
$$

where

$$
\eta(s, \not D)=\sum_{\lambda>0} \lambda^{-s}-\sum_{\lambda<0}(-\lambda)^{-s}
$$

Passing to the physical limit, ${ }^{1}$ we arrive to a simple expression for the parity anomaly [21]:

$$
W_{\text {odd }}=\lim _{s \rightarrow 0} \mu^{s} \Gamma(s) \zeta_{\text {odd }}(s, \not D)=\frac{\mathrm{i} \pi}{2} \eta(0, \not D) .
$$

This expression is quite general and further computational steps depend on a particular choice of boundary conditions and other aspects of the model. Fortunately, for our setup, which we described above, there is a suitable computational procedure, and in the forthcoming sections we explain how it works.

Before we go ahead, we would like to clarify, why we expect the parity anomaly to be different from zero, emphasising a peculiar role played by the boundary. In the standard situation, when the boundary is absent, the spectrum of $I D$ is symmetric with respect to 0 , hence there is no room for a parity anomaly. Indeed, since $\left\{\not D, \gamma^{5}\right\}=0$, for any eigenfunction $\psi_{\lambda}$, which corresponds to the eigenvalue $\lambda$, the quantity $\gamma^{5} \psi_{\lambda}$ is again an eigenfunction of $\not D$ but with the opposite eigenvalue. On the other side the boundary conditions break this property, since they do not respect chirality: $\Pi_{-} \gamma^{5}=\gamma^{5} \Pi_{+}$. In other words, in our setup the boundary conditions exhibit the only source of the spectral asymmetry.

\section{Gauge contribution}

Throughout this section we follow the reference ${ }^{2}[1]$. The spectral function $\eta(s, \not D)$ exhibits the following integral representation:

$$
\eta(s, \not D)=\frac{2}{\Gamma\left(\frac{s+1}{2}\right)} \int_{0}^{\infty} \mathrm{d} \tau \tau^{s} \operatorname{Tr}\left(\not D e^{-\tau^{2} \not D^{2}}\right)
$$

\footnotetext{
${ }^{1}$ Even though the regularised effective action (9) has a pole at $s=0$, its P-odd part is finite at the physical limit, since the root of the prefactor $\left(1-e^{-\mathrm{i} \pi s}\right)$ in Eq. (11) cancels this pole.

${ }^{2}$ In [1] the space-time was assumed to be flat, however, one can easily check that a presence of nontrivial curvature does not affect the variation of $W_{\text {odd }}$ over the gauge field. A three-dimensional prototype of this computation was previously presented in the book [23].
} 
Upon the variation of the gauge field

$$
A_{\mu}(x) \longrightarrow A_{\mu}(x)+\delta A_{\mu}(x)
$$

the $\eta$ function exhibits the variation

$$
\delta \eta(s, \not D)=\frac{2}{\Gamma\left(\frac{s+1}{2}\right)} \int_{0}^{\infty} d \tau \tau^{s} \frac{d}{d \tau} \operatorname{Tr}(\delta \not D) \tau e^{-\tau^{2} \not D^{2}},
$$

where

$$
\delta \not D=-\gamma^{\mu} \delta A_{\mu} .
$$

At the physical limit $s=0$ one can integrate over the parameter $\tau$ :

$$
\delta \eta(0, \not D)=-\frac{2}{\sqrt{\pi}} \lim _{t \rightarrow+0} \operatorname{Tr}(\delta \not D) \sqrt{t} e^{-t \not D^{2}}
$$

For an arbitrary matrix-valued function $Q$ the following asymptotic expansion holds at $t \longrightarrow+0$ :

$$
\operatorname{Tr} Q e^{-t \not D^{2}} \simeq \sum_{k=0}^{\infty} t^{\frac{k-4}{2}} a_{k}\left(Q, \not D^{2}\right)
$$

where the quantities $a_{k}$ are called "the heat kernel (or Seeley-DeWitt) coefficients", see [22] for a profound review. The structure of the heat kernel coefficients depends an a choice of boundary conditions. The heat kernel coefficients which arise from the local bag boundary conditions (8) were studied in [24]. In our case $a_{0}, a_{1}$ and $a_{2}$ vanish, therefore ${ }^{3}$

$$
\delta \eta(0, \not D)=-\frac{2}{\sqrt{\pi}} a_{3}\left(\delta \not D, \not D^{2}\right)=\left(-\frac{1}{4 \pi^{2}}\right) \sum_{\alpha} \int_{\partial \mathcal{M}_{\alpha}} d^{3} x \sqrt{h} \epsilon_{\alpha} \varepsilon^{n a b c}\left(\delta A_{a}\right) \partial_{b} A_{c}
$$

where $\varepsilon^{n a b c}$ stands for the Levi-Civita tensor. The variation of the parity-odd contribution to the one-loop effective action reads:

$$
\delta^{\text {gauge }} W_{\text {odd }}=\left(-\frac{\mathrm{i}}{8 \pi}\right) \sum_{\alpha} \int_{\partial \mathcal{M}_{\alpha}} d^{3} x \sqrt{h} \epsilon_{\alpha} \varepsilon^{n a b c}\left(\delta A_{a}\right) \partial_{b} A_{c} .
$$

If the gauge potential $A$ is defined globally on $\mathcal{M}$, we can recover the gauge contribution to $W_{\text {odd }}$ :

$$
W_{\text {odd }}^{\text {gauge }}=\frac{1}{4} \sum_{\alpha} \epsilon_{\alpha}\left(-\frac{\mathrm{i}}{4 \pi} \int_{\partial \mathcal{M}_{\alpha}} \sqrt{h} \varepsilon^{n a b c} A_{a} \partial_{b} A_{c}\right),
$$

where one can easily recognise the Chern-Simons action of the level 1/4.

It is important that there are other contributions to $W_{\text {odd }}$, which do not depend on the gauge field $A$. These terms involve the metric tensor and its derivatives, and we discuss this dependence in the next section. In conclusion we remark that the gauge contribution (22) does not depend on the metric tensor ${ }^{4}$.

\footnotetext{
${ }^{3}$ We refer to [1] for the computational details.

${ }^{4}$ We emphasise that $\varepsilon^{n a b c}$ is the Levi-Civita tensor rather than the Levi-Civita symbol.
} 


\section{Gravitational contribution}

Throughout this section we follow the reference ${ }^{5}$ [2]. The gravitational input enters in the Dirac operator through the vierbeins $e_{\mu}^{a}, a=0, . ., 3$, which appear in the gamma matrices $\gamma^{\mu}=e_{a}^{\mu} \gamma^{a}$ and in the spin connection $\omega$. Using again the integral representation Eq. (14) and considering the variation

$$
e_{a}^{\mu} \longrightarrow e_{a}^{\mu}+\delta e_{a}^{\mu}
$$

we arrive to (16), where

$$
\delta \not D=\mathrm{i} \gamma^{\mu} \delta \omega_{\mu}+\mathrm{i}\left(\delta e_{a}^{\mu}\right) \gamma^{a}\left(\nabla_{\mu}+\mathrm{i} A_{\mu}\right)
$$

is the 1-st order differential operator rather than the matrix valued function (c.f. Eq. (17)). At the physical limit $s=0$ we again obtain Eq. (18).

For the first order differential operator of the structure

$$
Q=Q_{1}^{\mu} \partial_{\mu}+Q_{0}
$$

where $Q_{1}^{\mu}$ and $Q_{0}$ are matrix valued functions, the asymptotic expansion at $t \longrightarrow+0$ has a slightly different structure with respect to Eq. (19):

$$
\operatorname{Tr} Q e^{-t D^{2}} \simeq \sum_{k=-1}^{\infty} t^{\frac{k-4}{2}} a_{k}\left(Q, \not D^{2}\right)
$$

There is a trick which allows to compute $a_{k}\left(Q, \not D^{2}\right)$ using the known expressions for $a_{k+2}(\tilde{Q}, \mathcal{L})$, where $\mathcal{L}$ is a generic Laplace-type operator and $\tilde{Q}$ is a generic matrix-valued function, see [2]. In our case $a_{-1}, a_{0}, a_{1}$ and $a_{2}$ vanish, therefore ${ }^{6}$

$$
\begin{aligned}
\delta^{\text {grav }} W_{\text {odd }} & =-\mathrm{i} \sqrt{\pi} a_{3}\left(\delta \not D, \not D^{2}\right)=\int_{\partial \mathcal{M}} d^{3} x \sqrt{h} \epsilon_{\alpha}\left\{-\frac{\mathrm{i}}{384 \pi}\left(\delta g_{j q}\right) \tilde{R}_{s p}{ }^{q k}: k \varepsilon^{n j s p}\right. \\
& \left.+\frac{\mathrm{i}}{256 \pi}\left(\left(\delta g_{s i}\right)_{; n} K_{p: l}^{i}-\left(\delta g_{s i}\right)\left(K_{l}^{i} K_{p: r}^{r}+K_{p}^{r} K_{l: r}^{i}+K^{r i} K_{r p: l}\right)\right) \varepsilon^{n s p l}\right\}
\end{aligned}
$$

where $^{7} \tilde{R}_{s p q k}$ stands for the Riemann tensor on the boundary, and $K_{i j}$ is the extrinsic curvature of the boundary. The semicolon and the colon denote the derivative with respect to Reimannian structures on $\mathcal{M}$ and $\partial \mathcal{M}$ respectively.

The solution of this variational equation reads:

$$
W_{\text {odd }}^{\text {grav }}=-\frac{\mathrm{i}}{4 \pi} \frac{1}{96} \int_{\partial \mathcal{M}} d^{3} x \sqrt{h} \epsilon_{\alpha}\left[\left(\widetilde{\Gamma}_{q i}^{r} \partial_{j} \widetilde{\Gamma}_{r k}^{q}+\frac{2}{3} \widetilde{\Gamma}_{q i}^{r} \widetilde{\Gamma}_{p j}^{q} \widetilde{\Gamma}_{r k}^{p}\right) \varepsilon^{n i j k}+\frac{3}{2} K_{s i} K_{p: l}^{i} \varepsilon^{n s p l}\right],
$$

where $\widetilde{\Gamma}_{q i}^{r}$ stand for the Christoffel symbols on the boundary. It is remarkable that apart from the gravitational Chern-Simons term (of the level 1/96) this answer contains a specific contribution of the extrinsic curvature. In conclusion we remark that Eq. (28) can be regarded as a parity odd boundary contribution to the Induced Gravity action. The induced gravity has been considered upon various regularisations [27], including the spectral one [28].

\footnotetext{
${ }^{5}$ In [2] the gauge potential $A$ was absent. Repeating the calculations of [2], one can easily demonstrate, that its presence does not affect the variation of $W_{\text {odd }}$ over the vierbeins.

${ }^{6}$ We refer to [2] for the computational details. Relevant results for heat kernel coefficients were first obtained in [25] and corrected in [26].

${ }^{7}$ Hereafter we put tildes over 3D geometric quantities to stress that they have to be computed with the induced boundary metric $h_{i j}$.
} 


\section{Comment on the classical symmetry}

Let us consider a symmetry of classical equations of motion

$$
\left\{\begin{array}{c}
\mathrm{i} \gamma^{\mu}\left(\frac{\partial}{\partial x^{\mu}}+\mathrm{i} A_{\mu}(x)\right) \psi=0 \\
\left.\frac{1}{2}\left(\mathbf{1}-\mathrm{i} \epsilon_{\alpha} \gamma^{5} \gamma^{n}\right) \psi\right|_{\partial \mathcal{M}}=0
\end{array}, \quad \epsilon_{\alpha} \in\{+1,-1\},\right.
$$

which is violated by quantum corrections Eq. (22) and Eq. (28).

The transformation (1), which was mentioned in the context of the parity anomaly on $\mathrm{R}^{3}$, is a bad candidate: if $x \in \mathcal{M}$ there is no guarantee that $-x \in \mathcal{M}$. The correct classical symmetry in a presence of boundaries is

$$
\left\{\begin{array}{c}
\psi(x) \longrightarrow \gamma^{5} \psi(x) \\
\epsilon_{\alpha} \longrightarrow-\epsilon_{\alpha}
\end{array}\right.
$$

This transformation inverts the nonzero spectrum of the Dirac operator, however it affects neither coordinates nor the gauge background.

\section{Discussions and outlook}

The gauge and the gravitational parity-violating contributions are given by Eq. (22) and Eq. (28) respectively.

It is remarkable that in both gauge and gravitational sectors the Chern-Simons actions appear with the coefficients, which are exactly twice smaller than the corresponding numbers in three-dimensional theories [3, 4, 18-20]. A naturalness of this ratio is clarified in $[1,2]$ considering Kaluza-Klein 3D limits of 4D manifolds.

A peculiar feature of the gravitational parity-violating terms Eq. (28) is a presence of the extrinsic curvature contribution $K_{i l} K_{k: j}^{l} \varepsilon^{n i j k}$ apart from the Chern-Simons term. The (bulk) Pontryagin type topological density

$$
\begin{aligned}
P_{\text {grav }} & =\frac{1}{4} \int_{\mathcal{M}} d^{4} x \sqrt{g} \varepsilon^{\mu \nu \alpha \beta} R_{\tau \mu \nu}^{\sigma} R_{\sigma \alpha \beta}^{\tau} \\
& =-\int_{\partial \mathcal{M}} d^{3} x \sqrt{h}\left[\left(\widetilde{\Gamma}_{i l}^{m} \partial_{j} \Gamma_{k m}^{l}+\frac{2}{3} \widetilde{\Gamma}_{i m}^{l} \widetilde{\Gamma}_{j p}^{m} \widetilde{\Gamma}_{k l}^{p}\right) \varepsilon^{n i j k}-2 K_{i l} K_{k: j}^{l} \varepsilon^{n i j k}\right]
\end{aligned}
$$

also contains both this combination and the Chern-Simon term, however the relative coefficient is different. Therefore Eq. (28) has no relation to the (bulk) Pontryagin type topological density, regardless of the choice of the sign factors $\varepsilon_{\alpha}$. From this point of view the situation in the gauge sector is different: if one chooses all $\varepsilon_{\alpha}$ equal to each other, all the gauge boundary contributions can be rewritten as a bulk integral of the Pontryagin density

$$
P_{\text {gauge }}=\frac{1}{4} \int_{\mathcal{M}} d^{4} x \sqrt{g} \varepsilon^{\mu v \alpha \beta} F_{\mu \nu} F_{\alpha \beta}=-\int_{\partial \mathcal{M}} d^{3} x \sqrt{h}\left(A_{i} \partial_{j} A_{k}\right) \varepsilon^{n i j k},
$$

where

$$
F_{\mu \nu}=\partial_{\mu} A_{\nu}-\partial_{v} A_{\mu}
$$

It is also remarkable that the parity violating terms are invariant upon the local Weyl transformation:

$$
g_{\mu v} \longrightarrow e^{2 \phi} g_{\mu v}
$$

The results (22) and (28) are not the end of the story, and they open various directions for future research. We worked with the Euclidean space-time, therefore one has to understand 
the Wick rotation procedure to the Lorentzian signature. Even if the boundary is absent, such a Wick rotation is a nontrivial task: the issue is analysed in details in the context of the Euclidean spectral geometry in [29]. A presence of boundaries makes the situation even more involved, since the Wick rotation must affect the boundary conditions as well.

Apart from that it is also interesting to generalise the results for the local bag boundary conditions with a nontrivial chiral phase (see e.g. [22]) and understand its physical role.

The parity anomaly is a purely spectral object, which comes out from the odd part of the $\zeta$ function (11). The third interesting problem in this context is to study a possible connection with the spectral action. The original spectral action is defined in [30] on manifolds without boundaries using a cutoff regularisation. Later on manifolds with boundaries [31-33] and the $\zeta$ function regularisation ${ }^{8}$ [34] (on manifolds without boundaries) have been considered within the spectral action approach as well. It would be very interesting to study a role played by the spectral asymmetry in the spectral action, which is based on the $\zeta$ function regularisation, as far as the boundary is concerned.

Acknowledgments. The author is grateful to Dmitri Vassilevich for collaboration on the basic projects [1] and [2] and related topics. This work is supported by the INFN Iniziativa Specifica GeoSymQFT.

\section{References}

[1] M. Kurkov and D. Vassilevich, Phys. Rev. D 96, no. 2, 025011 (2017)

[2] M. Kurkov and D. Vassilevich, JHEP 1803, 072 (2018)

[3] A. J. Niemi and G. W. Semenoff, Phys. Rev. Lett. 51, 2077 (1983).

[4] A. N. Redlich, Phys. Rev. Lett. 52, 18 (1984).

[5] M. Mulligan and F. J. Burnell, Phys. Rev. B 88, 085104 (2013)

[6] H. G. Zirnstein and B. Rosenow, Phys. Rev. B 88, no. 8, 085105 (2013)

[7] E. J. Konig, P. M. Ostrovsky, I. V. Protopopov, I. V. Gornyi, I. S. Burmistrov and A. D. Mirlin, Phys. Rev. B 90, no. 16, 165435 (2014)

[8] R. Wang, B. Wang and D. Y. Xing, arXiv:1705.06421 [cond-mat.str-el].

[9] O. Golan and A. Stern, Phys. Rev. B 98, no. 6, 064503 (2018)

[10] I. Fialkovsky, N. Khusnutdinov and D. Vassilevich, Phys. Rev. B 97, no. 16, 165432 (2018)

[11] C. T. Ma, arXiv:1802.08959 [hep-th].

[12] Y. Ferreiros and E. Fradkin, arXiv:1802.06087 [cond-mat.str-el].

[13] H. Fukaya, T. Onogi and S. Yamaguchi, Phys. Rev. D 96, no. 12, 125004 (2017)

[14] D. Vassilevich, JHEP 1807, 108 (2018)

[15] C. S. Chu and R. X. Miao, JHEP 1807, 005 (2018)

[16] R. X. Miao, arXiv:1806.10777 [hep-th].

[17] R. X. Miao, arXiv:1808.05783 [hep-th].

[18] M. A. Goni and M. A. Valle, Phys. Rev. D 34, 648 (1986).

[19] I. Vuorio, Phys. Lett. B 175, 176 (1986) [Erratum: Phys. Lett. B 181416 (1986)].

[20] J. J. van der Bij, R. D. Pisarski and S. Rao, Phys. Lett. B 179, 87 (1986).

[21] L. Alvarez-Gaume, S. Della Pietra and G. W. Moore, Annals Phys. 163, 288 (1985).

[22] D. V. Vassilevich, Phys. Rept. 388, 279 (2003)

\footnotetext{
${ }^{8}$ Even though the low momenta behaviours of the $\zeta$ function based spectral action [34] and the original cutoffbased spectral action [30] are the same, the high momenta behaviours are drastically different. In the cutoff-based approach the high momenta bosons do not propagate [35], what physically means that all gauge subgroups become strongly coupled at the energies above the cutoff scale. Such a "strong unification" has been considered beyond the scope of the spectral geometry, see e.g. $[36,37]$.
} 
[23] D. Fursaev and D. Vassilevich, Springer, Berlin, Germany (2011).

[24] V. N. Marachevsky and D. V. Vassilevich, Nucl. Phys. B 677, 535 (2004)

[25] T. P. Branson, P. B. Gilkey, K. Kirsten and D. V. Vassilevich, Nucl. Phys. B 563, 603 (1999)

[26] I. G. Moss, J. Phys. A 45, 374022 (2012)

[27] M. Visser, Mod. Phys. Lett. A 17, 977 (2002)

[28] M. A. Kurkov and M. Sakellariadou, JCAP 1401, 035 (2014)

[29] F. D’Andrea, M. A. Kurkov and F. Lizzi, Phys. Rev. D 94, 025030 (2016)

[30] A. H. Chamseddine and A. Connes, Commun. Math. Phys. 186, 731 (1997)

[31] A. H. Chamseddine and A. Connes, J. Geom. Phys. 61, 317 (2011)

[32] A. H. Chamseddine and A. Connes, Phys. Rev. Lett. 99, 071302 (2007)

[33] B. Iochum, C. Levy and D. Vassilevich, Commun. Math. Phys. 310, 367 (2012)

[34] M. A. Kurkov, F. Lizzi, M. Sakellariadou and A. Watcharangkool, Phys. Rev. D 91 no.6, 065013 (2015)

[35] M. A. Kurkov, F. Lizzi and D. Vassilevich, Phys. Lett. B 731, 311 (2014)

[36] V. A. Rubakov and S. V. Troitsky, hep-ph/0001213.

[37] A. A. Andrianov, D. Espriu, M. A. Kurkov and F. Lizzi, Phys. Rev. Lett. 111, no. 1, 011601 (2013) 